\title{
Protamine induced right ventricular dysfunction and systemic hypotension during transcatheter aortic valve replacement
}

\author{
Arsalan Hamid ${ }^{1}$, Mohammad Hashim Jilani ${ }^{2}$, Fahad Waqar ${ }^{2}$, David Lasorda ${ }^{3}$ \\ ${ }^{1}$ Department of Medicine, University of Mississippi Medical Center, Jackson, MS, United States \\ ${ }^{2}$ Division of Cardiovascular Health and Disease, University of Cincinnati College of Medicine, \\ Cincinnati, OH, United States \\ ${ }^{3}$ Department of Cardiology, Allegheny General Hospital, Pittsburgh, PA, United States
}

Presented herein, is the case of a 73-year-old male who underwent an uneventful transcatheter aortic valve replacement (TAVR) for severe non-rheumatic aortic valve stenosis. Protamine was administered intravenously to reverse anticoagulation, followed by large bore sheath removal. Immediately following protamine infusion, the patient developed profound hypotension with a systolic blood pressure of $60 \mathrm{mmHg}$. Transthoracic echocardiogram was being performed simultaneously to assess the prosthetic aortic valve which revealed a significant dilation of the right ventricle (RV) with reduction in RV systolic function (Fig. 1C, D). Clinical assessment of the patient ruled out arteriotomy site bleeding or valvular dysfunction as the cause of hypotension. Approximately 90 s later, the patient's hemodynamics recovered spontaneously without the use of vasopressors or any further intervention. Repeat echocardiogram revealed RV size and function had returned to baseline which were normal (Fig. 1A, B). While the precise mechanism of protamine induced systemic hypotension has not been determined, vasodilation or protamine induced pulmonary vasoconstriction secondary to an anaphylactic response may occur as a result of histamine release and increased nitric oxide production. This case presents a novel finding of protamine induced hypotension without features of shock observed during a TAVR procedure along with RV dilation that resolved spontaneously. The patient had a repeat echocardiogram at 3-month follow-up which showed normal biventricular size and function, normal pulmonary artery pressures and prosthetic valve function. We recommend that the RV should be adequately monitored during protamine administration and echocardiograms should be recorded before and after protamine administration to assess for sustained RV compromise.

Conflict of interest: None declared

\footnotetext{
Address for correspondence: Fahad Waqar, MD, Division of Cardiovascular Health and Disease, University of Cincinnati College of Medicine, 231 Albert B. Sabin Way, Cincinnati, OH 45267-0542, United States, tel: 513-558-4272, e-mail: fahad.waqar@uc.edu
}

Received: 10.02.2021 Accepted: 6.06.2021

This article is available in open access under Creative Common Attribution-Non-Commercial-No Derivatives 4.0 International (CC BY-NC-ND 4.0) license, allowing to download articles and share them with others as long as they credit the authors and the publisher, but without permission to change them in any way or use them commercially. 


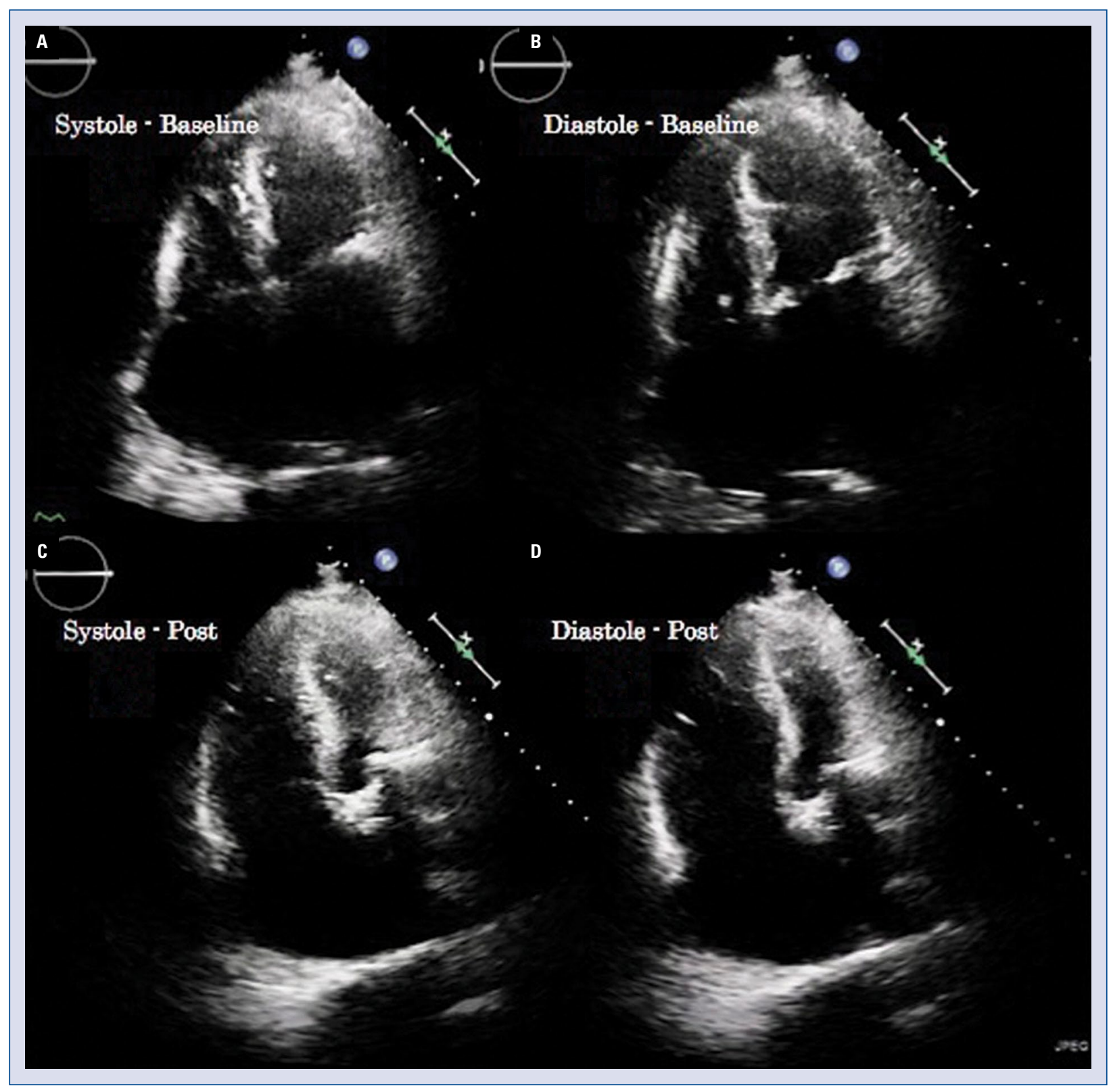

Figure 1. Transthoracic echocardiogram images at baseline (A, B) and after protamine administration (C, D). Image shows normal right ventricle (RV) size at baseline (A, B) and RV dilation after protamine administration (C, D). 